\title{
Online infrared thermography: Application to filament winding process defects detection
}

\author{
by $\underline{\text { O. COLAS }}$, B. COURTEMANCHE**, A. LE REUN**
}

*Nondestructive testing engineer, CETIM, Instrumentation engineering department, Route de la Jonelière, 44300, Nantes, France, oriane.colas@cetim.fr

${ }^{\star *}$ Composite process engineer, CETIM, Composite and Polymer engineering department, Chemin du Chaffault, 44340, Bouguenais, France, benoit.courtemanche@cetim.fr

\section{Abstract}

In the scope of composite process monitoring, the present publication will expose the implementation of infrared thermography on a filament winding pilot line. For that final purpose, infrared thermography has been evaluated, implemented and validated on the process line. Infrared thermography appears as a good asset for two purposes, detecting the defects created by the process and monitoring the process parameter evolution.

\section{Introduction}

Thermoplastic filament winding pilot line is composed of a robot head containing a laser and a filament tape feeding. The process consists in heating the surface of the composite tape in order to stick it to the surface of a liner. Several composite layers are made up to manufacture structures as pressure vessels or pipes. Due to the winding process, defects between the layers in the thickness or along the axe of the structure can appear. A CETIM project between composite engineering department and instrumentation engineering department has been carried out in order to identify the type, size, location of the critical defects that could occur during the process. The infrared thermography has then been qualified in order to integrate the process line as a control unit.

\section{Qualification}

\subsection{Defect implementation}

Two types of defects have to be detected following the information given by the composite process department. The first ones are the internal defects such as porosities and tape heterogeneities. The second are the winding defects which can be caused by a process operation mistake (wrong input), or an evolution of the machine behaviour. These two types of defects have been reproduced, with a certain gap with the reality for the internal defects but a good representation for the winding defects by biasing the process parameters.

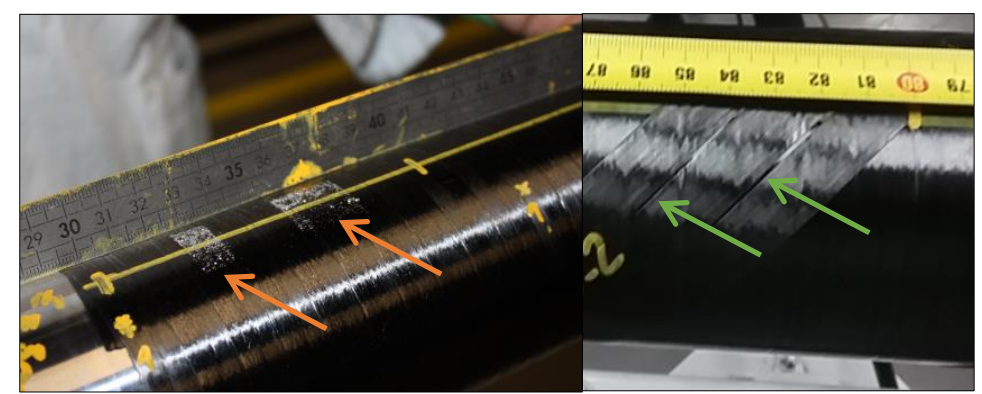

Fig. 1: Defect implementation (left: internal defects, right: winding defects).

\subsection{Sensitivity evaluation}

A comparison has been made between active pulsed thermography and passive thermography in order to select the most relevant technique, considering the sensitivity but also the operational aspects. Experimental studies have been both performed next to the pilot line, passive thermography during the process and active thermography after the production, the part remaining stable. Microscopic observations have then been carried out to characterize the shape and size of the defects. 
Passive thermography appears as the most relevant technique, giving a satisfying sensitivity, even though active thermography gives a better sensitivity and defect definition. The choice of passive thermography was made considering the higher flexibility in terms of implementation.

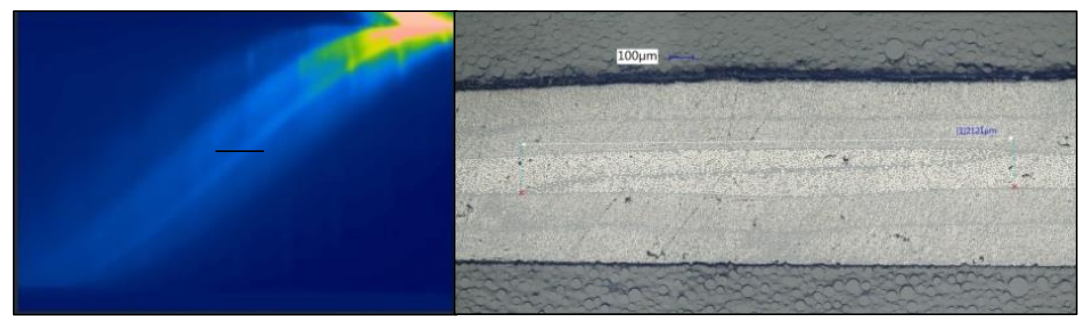

Fig. 2: Comparison between an infrared image obtained by passive thermography (left) and a micrographic analysis made along the black line of the infrared image (right).

\section{Adaptation to the production line}

\subsection{Mechanical tooling}

The first step of the implementation of infrared thermography on the pilot line was to design a tooling allowing the camera to be hold according to the process. The infrared camera has been implemented to the robot head taking into account the sizing of the machine and the moves occurring during the process, varying according the type of the structure in fabrication.

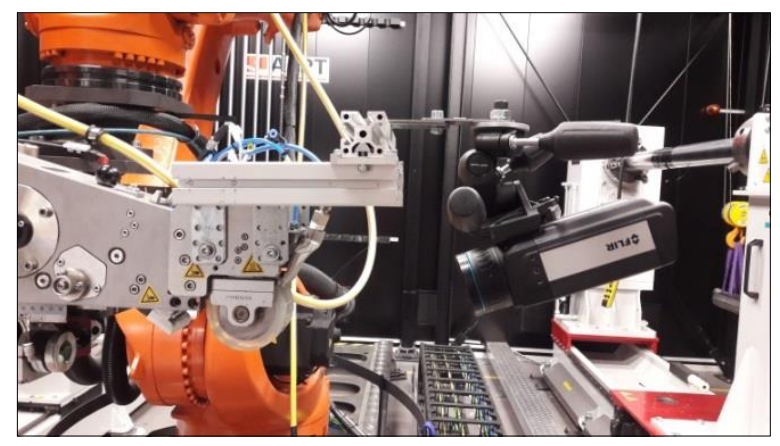

Fig. 3: Infrared camera mounted on the robot head.

\subsection{Validation on production}

Validation tests have been performed during a production at a speed of 7 meters $/ \mathrm{min}$. Winding defects such as gaps between tapes were detected. A post analysis tool has been developed in order to ease the thermal cartography analysis. On the Figure 4, gaps between tapes are enlightened in blue and overlaps in red.

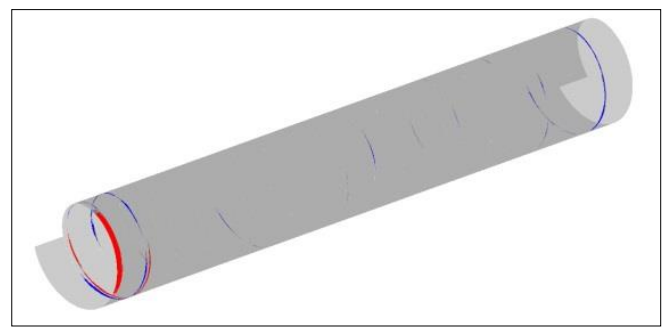

Fig. 4: 3D reconstruction of the infrared data.

\section{REFERENCES}

[1] Laurent Legrandjacques, Christophe Dehan, Jean-Claude Krapez, Francois Le Poutre. Method and device for the inspection of a material by thermal imaging. US patent, 2002 July 16.

[2] John A. Ritter, John F. Sjogren. Real-time infrared thermography inspection and control for automated composite material layup. US patent, 2006 August 31.

[3] Berend Denkena, Carsten Schmidt, Klaas Völtzer, Tristan Hocke. Thermographic online monitoring system for Automated Fiber Placement processes. In: Composites Part B: Engineering 2016; 97:239-243. 